\title{
On the multiplicativity of the linear combination of additive representation functions
}

\author{
Sándor Z. Kiss ${ }^{*}$ Csaba Sándor ${ }^{\dagger}$
}

\begin{abstract}
Let $A$ be a set of positive integers. For a fixed $k \geqslant 1$ and a positive integer $n$ let $R_{A, k}(n)$ denote the number of representations of $n$ as the sum of $k$ terms from the set $A$. In this paper we give a necessary and sufficient condition to the multiplicativity of the function $c_{1} R_{A, 1}(n)+c_{2} R_{A, 2}(n)$, where $c_{1}$ and $c_{2}$ are integers and $c_{2} \neq 0$.
\end{abstract}

2000 AMS Mathematics subject classification number: 11B34. Key words and phrases: additive number theory, representation functions, multiplicative function.

\section{Introduction}

Let $\mathbb{Z}^{+}$denote the set of positive integers. Let $A=\left\{a_{1}, a_{2}, \ldots\right\}, 0 \leqslant a_{1}<a_{2}<\ldots$ be an infinite sequence of nonnegative integers. Let $R_{A, k}(n)$ denote the number of solutions of the equation

$$
a_{i_{1}}+\ldots+a_{i_{k}}=n, \quad a_{i_{1}}, \ldots, a_{i_{k}} \in A
$$

where $n \in \mathbb{Z}^{+}$. In a series of papers P. Erdős, A. Sárközy and V. T. Sós studied the additive representation functions. In [2], [3], [4] they investigated the regularity properties, while [5] and [6] focused on the monotonicity of the additive representation functions. Grekos, Haddad, Helou and Pihko [7] proved that the representation function cannot be periodic. One can find some other results in surveys [11] and [12]. We say an arithmetic function $f(n)$ is multiplicative if $f(a b)=f(a) f(b)$ for every $a, b \in \mathbb{Z}^{+}$which are coprime. Obviously, if $f(n)$ is a multiplicative arithmetic function, then $f(1)=1$, while $R_{A, k}(1) \neq 1$. This implies that the additive representation function $R_{A, k}(n)$ cannot be multiplicative. Define the function $g(n)$ by

$$
g(n)=c_{1} R_{A, 1}(n)+c_{2} R_{A, 2}(n)+\ldots+c_{k} R_{A, k}(n),
$$

where $c_{1}, \ldots, c_{k}$ are integers. In this paper we focus on the multiplicativity of $g(n)$. As far as we know this function has not been investigated yet. In the case when $k=2$, i.e., for the representation function corresponding the two terms sums we give a necessary and sufficient condition for the multiplicativity of $g(n)$. In particular, we prove the following theorem.

\footnotetext{
*Institute of Mathematics, Budapest University of Technology and Economics, H-1529 B.O. Box, Hungary; This author was supported by the OTKA Grant No. NK105645. and K115288

${ }^{\dagger}$ Institute of Mathematics, Budapest University of Technology and Economics, H-1529 B.O. Box, Hungary, csandor@math.bme.hu. This author was supported by the OTKA Grant No. K109789. This paper was supported by the János Bolyai Research Scholarship of the Hungarian Academy of Sciences.
} 
Theorem 1. If $A \subset \mathbb{Z}^{+}$and $c_{1}, c_{2} \in \mathbb{Z}, c_{2} \neq 0$, then the function $g(n)=c_{1} R_{A, 1}(n)+$ $c_{2} R_{A, 2}(n)$ is multiplicative if and only if one of the following conditions holds: $A=\{1\}$ and $c_{1}=1$ or $A=\mathbb{Z}^{+}$and $c_{1}=c_{2}=1$ or $A=\left\{n^{2}: n \in \mathbb{Z}^{+}\right\}$and $c_{1}=c_{2}=1$.

Unfortunately we could not settle the case $k \geqslant 3$ thus it remains open.

Problem 1. When $k \geqslant 3$ what conditions on the set $A$ are needed to ensure that the function $g(n)$ is multiplicative?

Let $h(z)$ denote the generating function of the set $A$, i.e.,

$$
h(z)=\sum_{a \in A} z^{a}
$$

where $z=r e^{2 i \pi \alpha}$ and $r<1$, thus this infinite series is absolutely convergent. For a fixed $m$ nonzero integer consider the polynomial $p(z)=z+m z^{2}$. It is easy to see that $g(m)$ is the coefficient of $z^{m}$ in $p(h(z))$. In view of this observation Theorem 1 . asserts that for an infinitely set $A$ the function $g(n)$ is multiplicative if and only if $p(z)=z+z^{2}$ and set $A$ is the set of positive integers or the set of positive square numbers . However, our proof of Theorem 1. is elementary, the analytic approach may help to handle the general case when $k \geqslant 3$. On the other hand when $A=\mathbb{Z}^{+}$we give a full description of the multiplicativity. Let $S(n, k)$ denote the number of partitions of a set of $n$ elements into $k$ nonepty subsets. By convention, we write $S(0,0)=1$. The sequence of $S(n, k)$ is called Stirling numbers of the second kind [13].

Theorem 2. If $A=\mathbb{Z}^{+}$and $p(z)=\sum_{i=1}^{d} c_{i} z^{i}$ is a polynomial of degree $d$, then the function $g(n)$ is multiplicative if and only if $c_{i}=(i-1) ! \cdot S(d, i-1) \sum_{j=i-1}^{d}\left(\begin{array}{c}d-1 \\ j\end{array}\right)$.

\section{Proof of Theorem 1.}

First we prove the sufficiency. We denote the cardinality of a set $A$ by $|A|$. If $A=\{1\}$ and $c_{1}=1$, then it follows that $g(1)=1, g(2)=c_{2}$, and $g(n)=0$ if $n \geqslant 3$, thus $g(n)$ is multiplicative. In the next step when $A=\mathbb{Z}^{+}$and $c_{1}=c_{2}=1$, we get that

$$
g(n)=R_{A, 1}(n)+R_{A, 2}(n)=1+(n-1)=n,
$$

which is obviously multiplicative. In the last case when $A=\left\{n^{2}: n \geqslant 1, n \in \mathbb{N}\right\}$ and $c_{1}=c_{2}=1$ we use the formula for the number of representations of a positive integer as the sum of two squares [9]. This asserts that if the prime decomposition of $n$ is $n=2^{\alpha} \cdot p_{1}^{\alpha_{1}} \cdots p_{s}^{\alpha_{s}} \cdot q_{1}^{\beta_{1}} \cdots q_{t}^{\beta_{t}}$, where $p_{i} \equiv 1 \bmod 4$ and $p_{j} \equiv 3 \bmod 4$, for every $1 \leqslant i \leqslant s$ and $1 \leqslant j \leqslant t$, then

$$
\left|\left\{(x, y): x, y \in \mathbb{Z}, x^{2}+y^{2}=n\right\}\right|=\left\{\begin{array}{r}
4 \prod_{i=1}^{s}\left(\alpha_{i}+1\right), \text { if } t=0 \\
0, \text { if } t>0
\end{array} .\right.
$$

This implies that

$$
\left|\left\{(x, y): x, y \in \mathbb{Z}, x^{2}+y^{2}=n\right\}\right|=4\left|\left\{(x, y): x, y \in \mathbb{Z}^{+}, x^{2}+y^{2}=n\right\}\right|
$$




$$
+4\left|\left\{x: x \in \mathbb{Z}^{+}, x^{2}=n\right\}\right|=4 R_{A, 2}(n)+4 R_{A, 1}(n),
$$

thus we have

$$
g(n)=R_{A, 1}(n)+R_{A, 2}(n)=\left\{\begin{array}{r}
\prod_{i=1}^{s}\left(\alpha_{i}+1\right), \text { if } t=0 \\
0, \text { if } t>0
\end{array} .\right.
$$

Then it follows immediately that $g(n)$ is multiplicative. This proves the sufficient conditions.

In the next step we prove the other direction. Assume that the function $g(n)$ is multiplicative. As $A \subset \mathbb{Z}^{+}$, thus we have $R_{A, 2}(1)=0$, which implies that $g(1)=$ $c_{1} R_{A, 1}(1)+c_{2} R_{A, 2}(1)=c_{1} R_{A, 1}(1)$. For a multiplicative function $g(n)$ we know that $g(1)=1$. Thus we have $c_{1}=1$ and $R_{A, 1}(1)=1$, which implies that $1 \in A$. Let $A=\left\{a_{1}, a_{2}, \ldots\right\}$, where $1=a_{1}<a_{2}<\ldots$ Assuming that $k \neq l$, we have

$$
\begin{gathered}
g\left(a_{k}+a_{l}\right)=R_{A, 1}\left(a_{k}+a_{l}\right)+c_{2} R_{A, 2}\left(a_{k}+a_{l}\right) \\
=\left|\left\{i: a_{i}=a_{k}+a_{l}\right\}\right|+c_{2}\left|\left\{(i, j): a_{i}+a_{j}=a_{k}+a_{l}\right\}\right| .
\end{gathered}
$$

In the above formula we have $\left|\left\{i: a_{i}=a_{k}+a_{l}\right\}\right|=0$ or $\left|\left\{i: a_{i}=a_{k}+a_{l}\right\}\right|=1$ and $\left|\left\{(i, j): a_{i}+a_{j}=a_{k}+a_{l}\right\}\right| \geqslant 2$, thus we have

$$
g\left(a_{k}+a_{l}\right) \neq 0
$$

if $k \neq l$. In view of the fact that $a_{1}=1$, for $i>1$ we have

$$
g\left(a_{i}+1\right) \neq 0
$$

Proposition 1. If $A \neq\{1\}$, then $|A|=\infty$.

Proof. We prove by contradiction. Assume that $A \neq\{1\}$, but $|A|<\infty$. Let $a^{*}$ be the maximal element of the set $A$. If $a^{*}=2$, then $A=\{1,2\}$, thus we have $g(1)=1$, $g(2)=1+c_{2}, g(3)=2 c_{2}, g(4)=c_{2}$. It follows that $g(12)=g(3) \cdot g(4)=2 c_{2}^{2}$, but

$$
g(12)=\left|\left\{i: a_{i}=12, a_{i} \in A\right\}\right|+c_{2}\left|\left\{(i, j): a_{i}+a_{j}=12, a_{i}, a_{j} \in A\right\}\right|=0
$$

which is absurd. If $a^{*}>2$ we have two cases.

Case 1. $\frac{a^{*}}{2} \notin A$. Then we have

$$
g\left(a^{*}\right)=\left|\left\{i: a_{i}=a^{*}, a_{i} \in A\right\}\right|+c_{2}\left|\left\{(i, j): a_{i}+a_{j}=a^{*}, a_{i}, a_{j} \in A\right\}\right|,
$$

which implies that $2 \nmid g\left(a^{*}\right)$, thus $g\left(a^{*}\right) \neq 0$ and we get from $(2)$ that $g\left(a^{*}+1\right) \neq 0$. It follows that $g\left(a^{*}\left(a^{*}+1\right)\right)=g\left(a^{*}\right) \cdot g\left(a^{*}+1\right) \neq 0$. Since for every element of $A$ we have

$$
a_{i} \leqslant a^{*}<\frac{a^{*}\left(a^{*}+1\right)}{2}
$$

it follows that

$g\left(a^{*}\left(a^{*}+1\right)\right)=\left|\left\{i: a_{i}=a^{*}\left(a^{*}+1\right), a_{i} \in A\right\}\right|+2 c_{2}\left|\left\{(i, j): a_{i}+a_{j}=a^{*}\left(a^{*}+1\right), a_{i}, a_{j} \in A\right\}\right|=0$. 
Case 2. Assume that $\frac{a^{*}}{2} \in A$. As $a^{*}>2$ and in view of (2) it follows that $g\left(\frac{a^{*}}{2}+1\right) \neq 0$ and $g\left(a^{*}+1\right) \neq 0$. Since $2\left(\frac{a^{*}}{2}+1\right)-\left(a^{*}+1\right)=1$ it follows that $\frac{a^{*}}{2}+1$ and $a^{*}+1$ are obviusly coprime. Thus we have

$$
\left.g\left(\left(\frac{a^{*}}{2}+1\right)\left(a^{*}+1\right)\right)=g\left(\frac{a^{*}}{2}+1\right) \cdot g\left(a^{*}+1\right)\right) \neq 0 .
$$

On the other hand

$g\left(\left(\frac{a^{*}}{2}+1\right)\left(a^{*}+1\right)\right)=\left|\left\{i: a_{i}=\left(\frac{a^{*}}{2}+1\right)\left(a^{*}+1\right), a_{i} \in A\right\}\right|+\left|\left\{(i, j): a_{i}+a_{j}=\left(\frac{a^{*}}{2}+1\right)\left(a^{*}+1\right), a_{i}, a_{j} \in A\right\}\right|$.

Since

$$
a_{i} \leqslant a^{*}<a^{*}+1<\frac{\frac{a^{*}}{2}+1}{2}\left(a^{*}+1\right),
$$

which implies that $g\left(\left(\frac{a^{*}}{2}+1\right)\left(a^{*}+1\right)\right)=0$ a contradiction.

Let $p$ be a positive prime and $M_{p}=2^{p}-1$ denote a Mersenne prime. Let $F_{n}=2^{2^{n}}+1$ a Fermat number. In the next proposition we compute the possible values of $a_{2}$.

Proposition 2. If $A \neq\{1\}$, then $a_{2}=M_{p}$ or $a_{2}=8$ or $a_{2}=F_{m}-1$.

Proof. In the first step we prove that $a_{2}$ is a power of a prime, that is $a_{2}=p^{\alpha}, \alpha \geqslant 1$. Assume that $a_{2}>2$ and $a_{2}=u \cdot v$, where $u, v$ are coprime positive integers and $u, v>1$. It is clear that $u, v<a_{2}$ and $g\left(a_{2}\right)=1$. On the other hand $g\left(a_{2}\right)=g(u v)=g(u) \cdot g(v)$, thus $g(u) \neq 0$ and $g(v) \neq 0$, but conditions $1<w<a_{2}$ and $g(w) \neq 0$ imply $w=2$, contradiction. The same argument shows that $a_{2}+1$ is also a power of a prime. As $a_{2}$ and $a_{2}+1$ have different parity, one of them is a power of two. If $a_{2}=2^{n}$ and $a_{2}+1$ is a prime number, then $n=2^{m}$, that is $a_{2}=F_{m}-1$. If $a_{2}+1=p^{\alpha}, \alpha>1$, then it is well known [8] that the Catalan - equation $2^{n}+1=p^{\alpha}$ has the only solution $n=3, p=3$, $\alpha=2$. This implies that $a_{2}=8$. In the second case when $a_{2}+1=2^{n}$, then $a_{2}=2^{n}-1$, but $2^{n}-1 \neq p^{\alpha}$ when $\alpha>1$, thus $2^{n}-1$ is a Mersenne prime.

In the next proposition we study $a_{3}$.

Proposition 3. Let $a_{2}=M_{p}$. Then $a_{3}=8$ or $a_{3}=F_{m}-1$.

Proof. In the first step we prove that $a_{3}$ is a power of a prime. Assume that $a_{3}=u \cdot v$, where $u, v$ are coprime positive integers and $u, v>1$. If $n$ is a positive integer such that $g(n) \neq 0$ and $n<a_{3}$, then $n=2$ or $n=M_{p}$ or $n=M_{p}+1$ or $n=2 M_{p}$. This implies that $a_{3}=2 M_{p}$ or $a_{3}=M_{p}\left(M_{p}+1\right)$. We distinguish three cases.

Case 1. When $a_{3}=2 M_{p}$ and $a_{2}>3$, then it follows from (1) that $g\left(a_{3}+a_{2}\right) \neq 0$ and since $g(3)=0$ we have $g\left(a_{3}+a_{2}\right)=g\left(3 M_{p}\right)=g(3) g\left(M_{p}\right)=0$ which is absurd.

Case 2. If $a_{2}=3$ and $a_{3}=2 a_{2}=6$, then $g(2)=c_{2}, g(3)=1, g(6) \geqslant 1+c_{2}$ and $g(6)=g(2) g(3)$ a contradiction.

Case 3. When $a_{3}=M_{p}\left(M_{p}+1\right)$, then $g\left(a_{3}+a_{2}\right)=g\left(M_{p}\left(M_{p}+1\right)+M_{p}\right)=g\left(M_{p}\left(M_{p}+\right.\right.$ $2))=g\left(M_{p}\right) g\left(M_{p}+2\right)$. It is clear that if $n<a_{3}$ then $g(n) \neq 0$ when $n=1,2, M_{p}, M_{p}+$ $1,2 M_{p}$. Thus we have $g\left(a_{3}+a_{2}\right)=0$ which contradicts (1).

In the next step we prove that $a_{3}+1$ is a power of a prime similarly as above. We prove by contradiction. It follows that $a_{3}+1=2 M_{p}$ or $a_{3}+1=M_{p}\left(M_{p}+1\right)$. If $a_{3}+1=2 M_{p}$ 
and $p=2$ then $M_{2}=3$ and $a_{3}=5$. Thus $g(2)=c_{2}$ and $g(3)=1$, which implies that $g(6)=g(2) g(3)=c_{2}$. As $g(6)=R_{A, 1}(6)+c_{2} R_{A, 2}(6)=R_{A, 1}(6)+3 c_{2}=c_{2}$ so that $R_{A, 1}(6)=-2 c_{2}$ a contradiction. If $a_{2}=M_{p}>3$, then $a_{2} \geqslant 7$ and $a_{3}=2 M_{p}-1$. It follows from $(1)$ that $g\left(a_{3}+a_{2}\right) \neq 0$. As $M_{p} \equiv 7 \bmod 8$ it follows that $a_{3}+a_{2}=3 M_{p}-1 \equiv 4 \bmod 8$. Thus we have

$$
0 \neq g\left(a_{3}+a_{2}\right)=g\left(4\left(\frac{3 M_{p}-1}{4}\right)\right)=g(4) g\left(\frac{3 M_{p}-1}{4}\right) .
$$

Since $g(4)=0$, this is a contradiction. In the second case when $a_{3}=M_{p}\left(M_{p}+1\right)-1$, then

$$
g\left(a_{3}+a_{2}\right)=g\left(M_{p}^{2}+2 M_{p}-1\right)=g\left(2\left(M_{p}+\frac{M_{p}^{2}-1}{2}\right)\right)=g(2) g\left(M_{p}+\frac{M_{p}^{2}-1}{2}\right) .
$$

It follows from (1) that

$$
g\left(M_{p}+\frac{M_{p}^{2}-1}{2}\right) \neq 0 .
$$

On the other hand if $n<M_{p}^{2}+M_{p}-1=a_{3}$ and $g(n) \neq 0$ then $n=1,2, M_{p}, M_{p}+1,2 M_{p}$ a contradiction. A similar argument to the end of the proof of Proposition 2 gives that $a_{3}$ must be $M_{p^{\prime}}$ or 8 or $F_{m}-1$. If $a_{3}=M_{p^{\prime}}$, then $0 \neq g\left(a_{2}+a_{3}\right)=g\left(2^{p}+2^{p^{\prime}}-2\right)=$ $g(2) g\left(2^{p-1}+2^{p^{\prime}-1}-1\right)$, but conditions $1<w<a_{3}, g(w) \neq 0$ and $w$ is odd implies that $w=2^{p}-1$, a contradiction.

We may assume that $|A|=\infty$. We distiguish three cases.

Case 1. $c_{2}<0$. Then $g(n) \leqslant 1$. We have two subcases.

Case $1 a$. For every $p$ prime we have $g\left(p^{\alpha}\right) \in\{1,-1,0\}$.

In this case $g(n) \in\{1,-1,0\}$. It is clear that if $k \neq l$, then $a_{k}+a_{l} \in A$ since otherwise

$$
g\left(a_{k}+a_{l}\right)=R_{A, 1}\left(a_{k}+a_{l}\right)+c_{2} R_{A, 2}\left(a_{k}+a_{l}\right)=c_{2}\left|\left\{(i, j): a_{i}+a_{j}=a_{k}+a_{l}\right\}\right| \leqslant-2
$$

a contradiction. Thus we have $a_{1}+a_{2} \in A,\left(a_{1}+a_{2}\right)+a_{1} \in A$, which implies that

$g\left(2 a_{1}+2 a_{2}\right)=R_{A, 1}\left(2 a_{1}+2 a_{2}\right)+c_{2} R_{A, 2}\left(2 a_{1}+2 a_{2}\right) \leqslant 1-\left|\left\{(i, j): a_{i}+a_{j}=2 a_{1}+2 a_{2}\right\}\right| \leqslant-2$.

Since $2 a_{1}+2 a_{2}=\left(a_{1}+a_{2}\right)+\left(a_{1}+a_{2}\right)=\left(2 a_{1}+a_{2}\right)+a_{2}$ we get $g\left(2 a_{1}+2 a_{2}\right) \leqslant-2$ a contradiction.

Case $1 b$. There exists a prime $q$ such that $g\left(q^{\beta}\right)<-1$ for some $\beta \geqslant 1$. If $q \neq p$ prime and $g\left(p^{\alpha}\right) \leqslant-1$, then $g\left(p^{\alpha} q^{\beta}\right)=g\left(p^{\alpha}\right) g\left(q^{\beta}\right) \geqslant 2$ a contradiction. This implies that $g\left(p^{\alpha}\right) \in\{0,1\}$. We proved that if $k \neq l$, then $g\left(a_{k}+a_{l}\right) \neq 0$. We denote by $p^{\alpha}|| n$ if $p^{\alpha} \mid n$ but $p^{\alpha+1} \nmid n$. It follows that if $p$ is a prime such that $p^{\alpha} \| a_{k}+a_{l}, k \neq l$ then $g\left(p^{\alpha}\right) \neq 0$. We need the following lemma of Erdős and Turán [10].

Lemma 1. If $1 \leqslant a_{1}<a_{2}<\ldots<a_{12}$ integers, then the numbers $a_{k}+a_{l}$ has at least four prime divisors, that is there exist $p_{1}, p_{2}$, $p_{3}$ different primes, $p_{i} \neq q$ such that $p_{1}^{\alpha_{1}} \| a_{r}+a_{s}$, $p_{2}^{\alpha_{2}}\left\|a_{t}+a_{u}, p_{3}^{\alpha_{3}}\right\| a_{v}+a_{w}$, where $1 \leqslant r, s, t, u, v, w \leqslant 6, r \neq s, t \neq u, v \neq w$.

Then we have $g\left(p^{\alpha}\right) \in\{0,1\}$. As $g\left(p_{i}^{\alpha_{i}}\right) \neq 0$, thus we have $g\left(p_{i}^{\alpha_{i}}\right)=1$, which implies that $p_{i}^{\alpha_{i}} \in A$. It follows from (1) that $g\left(p_{i}^{\alpha_{i}}+p_{j}^{\alpha_{j}}\right) \leqslant-1$. Since $p_{i} \neq p_{j}$ and if $q \nmid n$, then $g(n) \geqslant 0$, it follows that $q\left|p_{1}^{\alpha_{1}}+p_{2}^{\alpha_{2}}, q\right| p_{1}^{\alpha_{1}}+p_{3}^{\alpha_{3}}, q \mid p_{2}^{\alpha_{2}}+p_{3}^{\alpha_{3}}$. Thus we have

$$
q \mid\left(p_{1}^{\alpha_{1}}+p_{2}^{\alpha_{2}}\right)+\left(p_{1}^{\alpha_{1}}+p_{3}^{\alpha_{3}}\right)-\left(p_{2}^{\alpha_{2}}+p_{3}^{\alpha_{3}}\right)=2 p_{1}^{\alpha_{1}} .
$$


It follows that $q \mid 2$, thus $q=2$ and $g(2 m+1) \in\{0,1\}$. If $a_{i}$ is even then it follows from (2) that $g\left(a_{i}+1\right) \leqslant-1$ a contradiction, which implies that $a_{i}$ is odd. Then it follows from Proposition 2. that $a_{2}=M_{p}=2^{p}-1$ and $a_{3}=8$ or $a_{3}=F_{m}-1$ by Proposition 3 , a contradiction. The proof of Case 1. is completed.

Case 2. $c_{2} \geqslant 2$. In view of Proposition 2. we have three possibilities for $a_{2}$. As $a_{1}=1$, if $a_{2}=F_{m}-1$ we have

$$
g\left(F_{m}\right)=g\left(a_{2}+1\right) \geqslant c_{2}\left|\left\{(i, j): a_{i}+a_{j}=a_{2}+1\right\}\right| \geqslant 2 c_{2},
$$

and

$$
g(2) \geqslant c_{2}\left|\left\{(i, j): a_{i}+a_{j}=2\right\}\right| \geqslant c_{2} .
$$

Thus we have $g\left(2 F_{m}\right)=g(2) g\left(F_{m}\right) \geqslant 2 c_{2}^{2}$. The quantity $2 F_{m}$ has the following three possible representations as the sum of two terms from the sequence $A$.

$$
2 F_{m}=1+\left(2 F_{m}-1\right)=\left(F_{m}-1\right)+\left(F_{m}+1\right)=F_{m}+F_{m} .
$$

We prove that $F_{m}+1$ cannot be contained in $A$. We prove by contradiction. Assume that $F_{m}+1 \in A$. If $m>0$ then we have

$$
g\left(F_{m}+1\right)=g\left(2^{2^{m}}+2\right)=g\left(2\left(2^{2^{m}-1}+1\right)\right)=g(2) g\left(2^{2^{m}-1}+1\right) .
$$

On the other hand $2<2^{2^{m}-1}+1 \leqslant 2^{2^{m}}-1<F_{m}$, thus we have $g\left(2^{2^{m}-1}+1\right) \neq 0$ a contradiction. If $m=0$, then we have $a_{1}=1, a_{2}=2, a_{3} \leqslant 4$, which implies that $g(2)=$ $c_{2}+1, g(3) \geqslant 2 c_{2}$ so that $g(6)=g(2) g(3) \geqslant\left(c_{2}+1\right) 2 c_{2}$. Clearly $6=1+5=2+4=3+3$, thus we have

$$
g(6) \leqslant 1+2 c_{2}+2 c_{2}+c_{2}=5 c_{2}+1<\left(c_{2}+1\right) 2 c_{2} \leqslant g(6)
$$

a contradiction. It follows that the quantity $2 F_{m}$ has the following two possible representations as the sum of two terms from the sequence $A$.

$$
2 F_{m}=1+\left(2 F_{m}-1\right)=F_{m}+F_{m} .
$$

Thus we have

$$
2 c_{2}^{2} \leqslant g\left(2 F_{m}\right) \leqslant 1+2 c_{2}+c_{2}=3 c_{2}+1,
$$

which is a contradiction if $c_{2} \geqslant 2$.

In the second case we assume that $a_{2}=8$. As $9=8+1=a_{2}+a_{1}$ we have $g(9) \geqslant 2 c_{2}$. It follows that $g(18)=g(9) g(2) \geqslant 2 c_{2} c_{2}=2 c_{2}^{2}$. It is clear that 18 has the following possible representations as the sum of two terms from $A$ : $1+17=8+10=9+9$. As $g(10)=g(2) g(5)$, if $10 \in A$, then $g(10) \neq 0$ and so $g(5) \neq 0$ a contradiction. Hence $g(18) \leqslant 1+2 c_{2}+c_{2}=1+3 c_{2}<2 c_{2}^{2} \leqslant g(18)$, a contradiction.

Thus $a_{2}=M_{p}$. It follows from Proposition 3. that $a_{3}=8$ or $a_{3}=F_{m}-1$. Assume that $a_{3}=8$. Then $a_{2}=3$ or $a_{2}=7$. If $a_{2}=3$, then $g(3)=1$ and $g(4)=2 c_{2}$, thus we have $g(12)=g(3) g(4)=2 c_{2}$. It is clear that 12 has the following possible representations as the sum of two terms from $A: 1+11=3+9$. If $9 \in A$ then clearly $a_{4}=9$. It follows that $g(10)=g(2) g(5)=g\left(a_{4}+a_{1}\right) \neq 0$, thus we have $g(5) \neq 0$ a contradiction. If $11 \in A$, then $g(14)=g(2) g(7)=g(3+11) \neq 0$, thus we have $g(7) \neq 0$ a contradiction. Thus 
$g(12) \leqslant 1$ which is a contradiction. If $a_{2}=7$, then $g\left(a_{2}+a_{3}\right)=g(15)=g(3) g(5) \neq 0$, which implies that $g(5) \neq 0$ a contradiction.

In the next case assume that $a_{3}=F_{m}-1, m \geqslant 1$. Then we have $g(2)=c_{2}$. We show that $g\left(F_{m}\right) \geqslant 2 c_{2}$. Clearly

$$
g\left(F_{m}\right)=\left|\left\{i: a_{i}=F_{m}\right\}\right|+c_{2}\left|\left\{(i, j): a_{i}+a_{j}=F_{m}\right\}\right| .
$$

Applying the fact that $F_{m}=1+\left(F_{m}-1\right)=a_{1}+a_{3}$, we obtain $g\left(F_{m}\right) \geqslant 2 c_{2}$ and therefore $g\left(2 F_{m}\right)=g(2) g\left(F_{m}\right) \geqslant 2 c_{2}^{2}$ we have $g\left(2 F_{m}\right) \geqslant 2 c_{2}^{2}$. The quantity $2 F_{m}$ has the following four possible representations as the sum of two terms from the sequence $A$.

$$
2 F_{m}=1+\left(2 F_{m}-1\right)=\left(F_{m}-1\right)+\left(F_{m}+1\right)=F_{m}+F_{m}=M_{p}+\left(F_{m}-M_{p}\right) .
$$

In the next step we prove that $g\left(2 F_{m}-1\right)=0$. We prove by contradiction. Assume that $g\left(2 F_{m}-1\right) \neq 0$. Then clearly $3 \mid 2 F_{m}-1$, i.e., $2 F_{m}-1=3^{u} v$, where $3 \nmid v$. For $m>1$ we know from Catalan's equation that $2 F_{m}-1=2^{2^{m}+1}+1 \neq 3^{u}$, thus $v>1$. It follows that $g\left(2 F_{m}-1\right)=g\left(3^{u}\right) g(v)$. We proved above that if $n$ is odd and $n<a_{3}$ and $g(n) \neq 0$, then $n=1$ or $n=M_{p}$ which is a contradiction. For $m=1$ we have $a_{2}=M_{p}=3$, $a_{3}=F_{m}-1=F_{1}-1=4$. Thus we have $g(2)=c_{2}, g(3)=1$, so that $g(6)=c_{2}$. It is clear that 6 has the following possible representations as the sum of two terms from $A$ : $6=1+5=3+3$. It follows that $5,6 \notin A$. Thus we have $g(4)=2 c_{2}+1, g(5)=2 c_{2}$. Thus we have $g(10)=g(2) g(5)=2 c_{2}^{2}$. It is clear that 10 has the following possible representations as the sum of two terms from $A: 10=1+9=3+7$. Since $c_{2} \mid g(10)$, thus $10 \notin A$ and $7,9 \in A$. An easy calculation shows that $g(7)=2 c_{2}+1$. Similarly we get that $g(12)=g(3) g(4)=2 c_{2}+1$, thus $11 \notin A$. Thus we have $g(14)=g(2) g(7)=c_{2}\left(2 c_{2}+1\right)$. It is clear that 14 has the following possible representations as the sum of two terms from A: $14=1+13=7+7$, which implies that $g(14) \leqslant 1+3 c_{2}$ a contradiction.

Equation $g\left(2 F_{m}-1\right) \neq 0$ implies that $2 F_{m}-1 \notin A$ and $F_{m} \notin A$ because $F_{m}-1 \in A$. We prove that $F_{m}+1 \notin A$. We prove by contradiction. Let us suppose that $F_{m}+1 \in A$, which implies $g\left(F_{m}+1\right) \neq 0$. On the other hand

$$
g\left(F_{m}+1\right)=g\left(2^{2^{m}}+2\right)=g\left(2\left(2^{2^{m}-1}+1\right)\right)=g(2) g\left(2^{2^{m}-1}+1\right),
$$

and $m>0$, thus we have $g\left(2^{2^{m}-1}+1\right) \neq 0$. We proved above that if $n$ is odd and $n<a_{3}=F_{m}-1$ and $g(n) \neq 0$, then $n=1$ or $n=M_{p}$. In the latter case we have $2^{2^{m}-1}+1=M_{p}=2^{p}-1$, which implies that $p=2, m=1$. In this case $a_{1}=1, a_{2}=3$ and $a_{3}=4$. We have already seen this case.

We get that the only possible representations of $2 F_{m}$ from $A 2 F_{m}=M_{p}+\left(F_{m}-M_{p}\right)$. It follows that $g\left(2 F_{m}\right) \leqslant 1+2 c_{2}$. As we proved above that $g\left(2 F_{m}\right) \geqslant 2 c_{2}^{2}$ we get a contradiction. The proof of Case 2. is completed.

Case 3. $c_{2}=1$. In this case we have $g(n)=R_{1, A}(n)+R_{2, A}(n)$. We prove that if $a_{2}=2$, then $A=\mathbb{Z}^{+}$. We distinguish two subcases.

Case 3a. Assume that $a_{2}=2, a_{3}=3$. We prove by induction that $a_{n}=n$ and $g(n)=n$. For $n \leqslant 3$ the statement is obvious. It follows from the well known Bertrand postulate that if $n \geqslant 3$ there exists an odd prime $p$ between $n / 2$ and $n$. Thus we get from the inductive step that $g(2 p)=g(2) g(p)=2 p$. On the other hand $g(2 p)=\mid\left\{i: a_{i}=\right.$ $2 p\}|+|\left\{(i, j): a_{i}+a_{j}=2 p\right\} \mid$. It is easy to see that $\left|\left\{(i, j): a_{i}+a_{j}=2 p\right\}\right| \leqslant 2 p-1$ and 
the equality holds if and only if $a_{i}=i$ for $i \leqslant 2 p-1$. This implies that if $i \leqslant 2 p$ then $a_{i}=i$. In our situation $2 p \geqslant n+1$, we obtain that $a_{n+1}=n+1$ and $g(n+1)=n+1$.

Case 3b. Assume that $a_{2}=2, a_{3}>3$. Then we have $g(2)=2$ and $g(3)=2$, thus we have $g(6)=g(2) g(3)=4=\left|\left\{i: a_{i}=6\right\}\right|+\left|\left\{(i, j): a_{i}+a_{j}=6\right\}\right|$. This implies that $a_{3}=4, a_{4}=5$ and $6 \notin A$. It follows that $g(4)=2, g(5)=3$. We get that $g(10)=g(2) g(5)=6=\left|\left\{i: a_{i}=10\right\}\right|+\left|\left\{(i, j): a_{i}+a_{j}=10\right\}\right|$. This implies that $8,9,10 \in A$. It is clear that 12 has the following possible representations as the sum of two terms from $A: 12=1+11=2+10=4+8=5+7$, and $g(12)=g(3) g(4)=4$, which implies that $7,11 \notin A$. Thus $g(7)=2$. It is clear that $g(14)=g(2) g(7)=4$ and 14 has the following representations as the sum of two terms from $A$ : $4+10=5+9$, which implies that $12,13,14 \notin A$. Obviously $g(15)=g(3) g(5)=6$, but counting the possible representation as the sum of two terms from set $A$ we get that $g(15) \leqslant 5$ which is a contradiction.

In the next step we prove that if $a_{2}>2$ then $a_{2}=4$. We have two subcases.

Case 3c. Assume that $a_{2}=3$. Then we have $g(2)=1, g(3)=1$. As $g(6)=g(2) g(3)=$ 1 , thus we have $5,6 \notin A$. If $4 \notin A$, we get that $g(4)=2, g(5)=0$, which implies that $g(10)=g(2) g(5)=0$ thus we have $7,9,10 \notin A$. We get that $g(7)=0$, which implies that $g(14)=g(2) g(7)=0$. This gives that $11,13,14 \notin A$. As $g(12)=g(3) g(4)=2$ and in view of the above facts we obtain that $g(12) \leqslant 1$ which is a contradiction. Thus we get that $a_{3}=4, g(4)=3, g(5)=2$. Assume that $9 \in A$. Then we have from $10=1+9$ that $g(10)=g(2) g(5)=2$, so $7,10 \notin A$, and $g(7)=2$. It follows that $g(12)=g(3) g(4)=3$ and $12=3+9$, thus we have $8,11 \notin A$ and $12 \in A$. In view of $g(14)=g(2) g(7)=2$, thus $13 \in A$ and $14 \notin A$. The representation $21=9+12$ and $g(21)=g(3) g(7)=2$ imply that $17,18,20,21 \notin A$. It is clear that 20 has the following possible representations as the sum of two terms from $A$ : $20=1+19=4+16$, and $g(20)=g(4) g(5)=6$, which implies that $g(20) \leqslant 4$ which is a contradiction. We get that $9 \notin A$. Because of $4 \in A$, we have $g(1)=1, g(2)=1, g(3)=1, g(4)=3$, therefore $g(6)=g(2) g(3)=1$, thus $5,6 \notin A$, and $g(5)=2$. In view of $g(10)=g(2) g(5)=2$ we obtain that $7 \in A$ and $10 \notin A$. As $g(12)=g(3) g(4)=3$, thus we have $12 \in A$. It is clear that $g(7)=3$. Since $g(15)=g(3) g(5)=2$, we have $8,11,14,15 \notin A$, and therefore $g(12) \leqslant 1$, which is absurd. Thus we have $a_{2} \neq 3$.

Case 3d. Assume that $a_{2}>4$. We know that $a_{2}=F_{m}-1$ or $a_{2}=M_{p}$ or $a_{2}=8$.

Let us suppose that $a_{2}=F_{m}-1, m \geqslant 2$. Then we have $g\left(a_{2}+a_{1}\right)=g\left(F_{m}\right) \geqslant 2$ and $g\left(2 F_{m}\right)=g(2) g\left(F_{m}\right) \geqslant 2$. But the possible representations as the sum of two terms of $A$ are $2 F_{m}=\left(F_{m}-1\right)+\left(F_{m}+1\right)=F_{m}+F_{m}=1+\left(2 F_{m}-1\right)$. If $F_{m}+1 \in A$, then

$$
g\left(F_{m}+1\right)=g\left(2^{2^{m}}+2\right)=g\left(2\left(2^{2^{m}-1}+1\right)\right)=g(2) g\left(2^{2^{m}-1}+1\right) \neq 0,
$$

thus $g\left(2^{2^{m}-1}+1\right) \neq 0$, a contradiction, which implies that $F_{m}+1 \notin A$. We show that $g\left(2 F_{m}-1\right)=0$. Suppose that $g\left(2 F_{m}-1\right)>0$. It is clear that $3 \mid 2 F_{m}-1$ and so $2 F_{m}-1=3^{\alpha} \cdot t$, where $t>1$ and $3 \nmid t$. It follows that

$$
g\left(2 F_{m}-1\right)=g\left(3^{\alpha} \cdot t\right)=g\left(3^{\alpha}\right) \cdot g(t) \neq 0,
$$

thus we have $g\left(3^{\alpha}\right) \neq 0, g(t) \neq 0$ a contradiction. We obtain that $F_{m}, 2 F_{m}-1 \notin A$, because $2 F_{m}-1=\left(F_{m}-1\right)+F_{m}$. Thus $g\left(2 F_{m}\right) \leqslant 1$ which is absurd. This implies that $a_{2} \neq F_{m}-1$. 
Assume that $a_{2}=8$. Then we have $g(9)=g(1+8) \geqslant 2$, therefore $g(18)=g(2) g(9) \geqslant 2$ and we have three possibilities to write integer 18 as the sum of two terms from $\mathrm{A}$ as $18=1+17=9+9=8+10$. If $9 \in A$ or $10 \in A$, then $g(10) \neq 0$. Hence we have $g(10)=g(2) g(5) \neq 0$, thus $g(5) \neq 0$ a contradiction. It follows that $17 \in A$. Then we have $g(8+17)=g(25) \geqslant 2$, thus $g(50) \geqslant 2$. As $g(3)=g(4)=g(5)=g(7)=0$, and for every decomposition $50=k+(50-k), 1 \leqslant k \leqslant 25$ one can find a prime power $p^{\alpha} \in\{3,4,5,7\}$ and an integer $l \in\{0,1,8,17\}$ such that $p^{\alpha} \| k+l$ or $p^{\alpha}|| 50-k+l$ we get that either $g(k)=0$ or $g(50-k)=0$, therefore either $k \notin A$ or $50-k \notin A$, thus $g(50) \leqslant 1$, contradiction.

It follows that $a_{2}=M_{p}$. By Proposition 3 we have $a_{3}=F_{m}-1$ or $a_{3}=8$. If $a_{3}=F_{m}-1$, then $g\left(2^{2^{m}}+1\right) \geqslant 2$, hence $g\left(2^{2^{m}+1}+2\right)=g(2) g\left(2^{2^{m}}+1\right) \geqslant 2$. It follows that the possible representations of $2^{2^{m}+1}+2$ are the following

$2^{2^{m}+1}+2=1+\left(2^{2^{m}+1}+1\right)=2^{p}-1+\left(2^{2^{m}+1}-2^{p}+3\right)=2^{2^{m}}+\left(2^{2^{m}}+2\right)=\left(2^{2^{m}}+1\right)+\left(2^{2^{m}}+1\right)$.

It is clear that if $g\left(2^{2^{m}+1}+1\right) \neq 0$, then $2^{2^{m}+1}+1=3^{\alpha} t, 3 \nmid t, t>1$ (now $m>1$ ), therefore $g\left(3^{\alpha}\right) \neq 0$ and $g(t) \neq 0$, which is absurd, because only for one odd $w, 1<w<a_{3}$ holds $g(w) \neq 0$. This implies that $2^{2^{m}}+1 \notin A$ and $2^{2^{m}+1}+1 \notin A$. If $2^{2^{m}+1}-2^{p}+3 \in A$ then we have $g\left(\left(2^{2^{m}+1}-2^{p}+3\right)+1\right)=g(4) g\left(2^{2^{m}-1}-2^{p-2}+1\right) \neq 0$, thus $g(4) \neq 0$ which is absurd. In the last case if $2^{2^{m}}+2 \in A$ then we have $g\left(2^{2^{m}}+2\right)=g(2) g\left(2^{2^{m}-1}+1\right) \neq 0$, thus $g\left(2^{2^{m}-1}+1\right) \neq 0$ which is impossible. Hence $g\left(2^{2^{m}+1}+2\right) \leqslant 1$, a contradiction. This implies that $a_{2}=4$.

In the next step we prove that $a_{3}=9$. If $a_{3}=5$, then $g(2)=1, g(3)=0$ and $2 \leqslant g(6)=g(2) g(3)=0$ a contradiction. If $a_{3}=6$, then $g(2)=2, g(3)=0$ and $1=g(6)=g(2) g(3)=0$ a contradiction. If $a_{3}=7$, then $g(3)=0$ and $g(9 k+3)=$ $g(3(3 k+1))=g(3) g(3 k+1)=0$, thus $9 k+3 \notin A$. On the other hand $g(9 k+6)=$ $g(3(3 k+2))=g(3) g(3 k+2)=0$, thus $9 k+6 \notin A$. It is clear that if $9 k+2 \in A$, then $9 k+3=(9 k+2)+1$, thus $g(9 k+3) \geqslant 2$ which is impossible. It is easy to see similarly that $9 k-1,9 k-4 \notin A$. This implies that $3 k+2 \notin A$, specially $8 \notin A$. The equality $g(10)=g(2) g(5)=2$ implies $9 \in A$ and $10 \notin A$. Then $g(14)=g(2) g(7)=1$, which implies that $13 \notin A$. We know $g(18)=g(2) g(9)=1$, which implies that $18 \notin A$. Thus we have $g(40)=g(5) g(8)=2 \cdot 3=6$, but $g(40) \leqslant 5$, because the possible representation as the sum of two terms from the set $A$ are $40=4+36=9+31$. If $a_{3}=8$, then $g(12)=g(3) g(4)=0$, but $12=4+8,4,8 \in A$, therefore $g(12)>0$, which is absurd. On the other hand if $a_{3} \geqslant 10$, then $1 \geqslant g(10)=g(2) g(5)=1 \cdot 2=2$ which is impossible. It follows that $a_{3}=9$.

In the next step we will prove that $a_{4}=16$. Assume that the first three elements of $A$ are $1,4,9 \in A$. For the fourth element of $A$ we have seven possibilities. If $10 \in A$, then $3=g(10)=g(2) g(5)=2$ which is absurd. If $11 \in A$, then $2 \leqslant g(12)=g(3) g(4)=0$ which is absurd. If $12 \in A$, then $1 \leqslant g(12)=g(3) g(4)=0$ which is absurd. If $13 \in A$, then $2 \leqslant g(14)=g(2) g(7)=0$ which is absurd. If $14 \in A$, then $1=g(14)=g(2) g(7)=0$ which is absurd. If $15 \in A$, then $1=g(15)=g(3) g(5)=0$ which is absurd. On the other hand if $a_{4} \geqslant 17$, then $g(20)=g(4) g(5)=2$, then $19 \in A$, thus $2 \leqslant g(28)=g(4) g(7)=0$ which is impossible. It follows that $a_{4}=16$.

In the next step we will prove that $a_{5}=25$. Assume that the first four elements of $A$ are $1,4,9,16 \in A$. For the fifth element of $A$ we have nine possibilities. If $17 \in A$, then $2 \leqslant g(21)=g(3) g(7)=0$ which is absurd. If $18 \in A$, then $2 \leqslant g(22)=g(2) g(11)=0$ 
which is absurd. If $19 \in A$, then $4 \leqslant g(20)=g(4) g(5)=2$ which is absurd. If $20 \in A$, then $3=g(20)=g(4) g(5)=2$ which is absurd. If $21 \in A$, then $1=g(21)=g(3) g(7)=0$ which is absurd. If $22 \in A$, then $1=g(22)=g(2) g(11)=0$ which is absurd. If $23 \in A$, then $2 \leqslant g(24)=g(3) g(8)=0$ which is absurd. If $24 \in A$, then $1=g(24)=g(3) g(8)=0$ which is absurd. On the other hand if $a_{5} \geqslant 26$, then $1 \geqslant g(26)=g(2) g(13)=2$ which is impossible. It follows that $a_{5}=25$.

We will prove that the $n$th element of $A$ is $a_{n}=n^{2}$ for every $n$.

Assume that the first five elements of $A$ are $a_{1}=1, a_{2}=4, a_{3}=9, a_{4}=16$ and $a_{5}=25$. It is clear that If $26 \in A$, then $3=g(26)=g(2) g(13)=2$ which is absurd. If $27 \in A$, then $2 \leqslant g(28)=g(4) g(7)=0$ which is absurd. If $28 \in A$, then $1=g(28)=g(4) g(7)=0$ which is absurd. If $29 \in A$, then $2 \leqslant g(30)=g(3) g(10)=0$ which is absurd. If $30 \in A$, then $1=g(30)=g(3) g(10)=0$ which is absurd. If $31 \in A$, then $2 \leqslant g(35)=g(5) g(7)=0$ which is absurd.

Let us suppose that $a_{1}=1, a_{2}=4, \ldots, a_{n}=n^{2}$ for $n \geqslant 5$ and $a_{n+1} \geqslant 32$. We prove that $a_{n+1}=(n+1)^{2}$. We have two cases.

Case 1. $a_{n+1}<(n+1)^{2}$. We prove the following proposition.

Proposition 4. If $a_{n+1}$ and $a_{n+1}+1$ are both prime power and $a_{n+1}$ is not a square, $a_{n+1} \geqslant 32$, then $(n+1)^{2}-a_{n+1}>16$.

Proof. It is clear that one of $a_{n+1}$ and $a_{n+1}+1$ is even, thus we have two possibilities. If $a_{n+1}=2^{\alpha}$, so that $\alpha$ is odd, thus $3 \mid a_{n+1}+1=2^{\alpha}+1$. It follows that $2^{\alpha}+1=3^{\beta}$. This implies that $\alpha=3$ and $a_{n+1}=8$ a contradiction. If $a_{n+1}+1=2^{\alpha}$, but $a_{n+1}$ is a power of a prime, thus $2^{\alpha}-1=p^{\gamma}$. It follows from [8] that $a_{n+1}$ is a prime, then we have $a_{n+1}=M_{p}$, where $p \geqslant 7$. It is easy to see that $2^{p}+1 \neq(n+1)^{2}, 2^{p}+4 \neq(n+1)^{2}$ and $2^{p}+9 \neq(n+1)^{2}$, thus we have $2^{p}+16 \leqslant(n+1)^{2}$, therefore $(n+1)^{2}-a_{n+1} \geqslant 17$.

In the next step we show that both $a_{n+1}$ and $a_{n+1}+1$ must be a power of a prime. Assume contrary that $a_{n+1}=u \cdot v$, where $u$ and $v$ are coprime positive integers and $u$, $v>1$. Let $G(k)$ denote that values of $g(k)$ which are corresponding to the set of squares. Then we have

$$
g\left(a_{n+1}\right)=g(u) g(v)=G(u) G(v)=G\left(a_{n+1}\right) .
$$

On the other hand

$g\left(a_{n+1}\right)=1+\left|\left\{(i, j): a_{i}+a_{j}=a_{n+1}, i, j \leqslant n\right\}\right|=1+\left|\left\{(i, j): i^{2}+j^{2}=a_{n+1}\right\}\right|=1+G\left(a_{n+1}\right)$,

which is a contradiction. Assume that $a_{n+1}+1=u \cdot v$, where $u$ and $v$ are coprime positive integers and $u, v>1$. Then we have

$$
g\left(a_{n+1}+1\right)=g(u v)=g(u) g(v)=G(u) G(v)=G\left(a_{n+1}+1\right) .
$$

On the other hand

$$
g\left(a_{n+1}+1\right) \geqslant 2+\left|\left\{(i, j): i^{2}+j^{2}=a_{n+1}+1\right\}\right|
$$

and

$$
G\left(a_{n+1}+1\right) \leqslant 1+\left|\left\{(i, j): i^{2}+j^{2}=a_{n+1}+1, i, j \leqslant n\right\}\right|,
$$


which is absurd. We have already shown that if both $a_{n+1}$ and $a_{n+1}+1$ are a power of a prime and $a_{n} \geqslant 32$, then $a_{n+1}<(n+1)^{2}-16$. It is clear that there are exactly two even numbers among $a_{n+1}, a_{n+1}+1, a_{n+1}+4, a_{n+1}+9$ and neither of them are a power of 2 , i.e., $a_{n+1}+l^{2}=2^{\alpha} v$, where $v>1$ odd and $0 \leqslant l \leqslant 3$. Then we have

$$
g\left(a_{n+1}+l^{2}\right)=g\left(2^{\alpha} v\right)=g\left(2^{\alpha}\right) g(v)=G\left(2^{\alpha}\right) G(v)=G\left(a_{n+1}+l^{2}\right) .
$$

On the other hand

$g\left(a_{n+1}+l^{2}\right) \geqslant 1+\left|\left\{(i, j): i^{2}+j^{2}=a_{n+1}+l^{2}\right\}\right|>\left|\left\{(i, j): i^{2}+j^{2}=a_{n+1}+l^{2}\right\}\right|=G\left(a_{n+1}+l^{2}\right)$,

which is impossible. The proof of Case 1. is completed.

Case 2. $a_{n+1}>(n+1)^{2}$. It is clear that there are exactly two even numbers among $(n+1)^{2},(n+1)^{2}+1$. We have two possibilities. If $(n+1)^{2}$ is even, but not a power of 2 , then $(n+1)^{2}=u \cdot v$, where $u, v>1$ coprime positive integers. Then we have

$$
g\left((n+1)^{2}\right)=g(u v)=g(u) g(v)=G(u) G(v)=G\left((n+1)^{2}\right) .
$$

On the other hand

$G\left((n+1)^{2}\right)=1+\left|\left\{(i, j): i^{2}+j^{2}=(n+1)^{2}, i, j>0\right\}\right|>\left|\left\{(i, j): i^{2}+j^{2}=(n+1)^{2}, i, j>0\right\}\right|=g(n+1)$,

which is impossible. If $(n+1)^{2}+1$ is even, i.e., $(n+1)^{2}+1=2 v$, where $2 \nmid v$.

$$
g\left((n+1)^{2}+1\right)=g(2 v)=g(2) g(v)=G(2) G(v)=G\left((n+1)^{2}+1\right) .
$$

On the other hand

$$
\begin{aligned}
& G\left((n+1)^{2}+1\right)=\left|\left\{(i, j): i^{2}+j^{2}=(n+1)^{2}+1, i, j>0\right\}\right|= \\
& 2+\left|\left\{(i, j): i^{2}+j^{2}=(n+1)^{2}+1,1 \leqslant i, j \leqslant n+1\right\}\right|> \\
& 1+\left|\left\{(i, j): i^{2}+j^{2}=(n+1)^{2}+1,1 \leqslant i, j \leqslant n\right\}\right| \geqslant g\left((n+1)^{2}+1\right) \\
& >2+\left|\left\{(i, j): i^{2}+j^{2}=(n+1)^{2}+1,1 \leqslant i, j \leqslant n+1\right\}\right| \geqslant 1+\left|\left\{(i, j): i^{2}+j^{2}=(n+1)^{2}+1,1 \leqslant i, j \leqslant n+1\right\}\right| \geqslant \\
& G\left(2^{2 m}+4\right)=G\left(4\left(1+2^{2 m-2}\right)\right)=G(4) G\left(1+2^{2 m-2}\right)=g(4) g\left(1+2^{2 m-2}\right)=g\left(2^{2 m}+4\right) . \\
& G\left(2^{2 m}+4\right)=2+\left|\left\{(i, j): i^{2}+j^{2}=2^{2 m}+4,0 \leqslant i, j<2^{m}\right\}\right|=g\left(2^{2 m}+4\right),
\end{aligned}
$$

therefore $2^{2 m}+3 \in A$ Thus we have

$$
g\left(2^{2 m}+12\right)=g\left(4\left(3+2^{2 m-2}\right)\right)=g(4) g\left(3+2^{2 m-2}\right)=G(4) G\left(3+2^{2 m-2}\right)=G\left(2^{2 m}+12\right) .
$$

On the other hand

$$
G\left(2^{2 m}+12\right)=\left|\left\{(i, j): i^{2}+j^{2}=2^{2 m}+12,0<i, j<2^{m}\right\}\right|
$$

but

$$
g\left(2^{2 m}+12\right)=2+\left|\left\{(i, j): i^{2}+j^{2}=2^{2 m}+12,0<i, j<2^{m}\right\}\right|,
$$

which is impossible. 


\section{Proof of Theorem 2.}

It is easy to see that

$$
\begin{gathered}
p(g(z))=p\left(\frac{z}{1-z}\right)=c_{1} \frac{z}{1-z}+c_{2}\left(\frac{z}{1-z}\right)^{2}+\ldots+c_{d}\left(\frac{z}{1-z}\right)^{d} \\
= \\
c_{1} \sum_{k=1}^{\infty}\left(\begin{array}{c}
k-1 \\
1-1
\end{array}\right) z^{k}+c_{2} \sum_{k=2}^{\infty}\left(\begin{array}{c}
k-1 \\
1
\end{array}\right) z^{k}+c_{3} \sum_{k=3}^{\infty}\left(\begin{array}{c}
k-1 \\
2
\end{array}\right) z^{k}+\ldots+c_{d} \sum_{k=t}^{\infty}\left(\begin{array}{c}
k-1 \\
d-1
\end{array}\right) z^{k} .
\end{gathered}
$$

As $g(k)=\sum_{s=1}^{d} c_{i}\left(\begin{array}{c}k-1 \\ s-1\end{array}\right)$ if $c_{t}<0$, then $g(k)<0$,fFor $k \geqslant k_{0}$, then for $p, q>k_{0}$, where $(p, q)=1$, then we have $g(p)<0, g(q)<0$ which implies that $0>g(p q)=g(p) g(q)>0$ a contradiction. It follows that $c_{d}>0$. This implies that there exists $k_{0}$ such that for $k>k_{0}$ we have $g(k)>0$. In fact, for every $k \in \mathbb{Z}^{+}, g(k)>0$, because for prime number $p>k_{0}$ and $p>k$ we have $g(k p)=g(p) g(k)>0$, and $g(p)>0$, which implies that $g(k)>0$. It is clear that there exists $k \geqslant k_{1}$ such that $g(k)$ is monotonous increasing. As $g(k)$ is multiplicative, then $\log g(k)$ is additive and $\lim \inf (\log g(k+1)-\log g(k)) \geqslant 0$, then it follows form a well known theorem of Erdös [1] then $\log g(k)=d \log k$, where $d$ is a positive constant. Thus we have $g(k)=k^{d-1}$. On the other hand

$$
(n-1)^{d}=\sum_{i=1}^{d}\left(\begin{array}{c}
n-1 \\
i-1
\end{array}\right)(i-1) ! \cdot S(d-1, i-1) .
$$

Thus we have

$$
\begin{gathered}
n^{d-1}=(n-1+1)^{d-1}=\sum_{j=0}^{d-1}\left(\begin{array}{c}
d-1 \\
j
\end{array}\right)(n-1)^{j} \\
=\sum_{j=0}^{d-1}\left(\begin{array}{c}
d-1 \\
j
\end{array}\right) \sum_{i=1}^{j+1}\left(\begin{array}{c}
n-1 \\
i-1
\end{array}\right)(i-1) ! \cdot S(d-1, i-1)=\sum_{i=1}^{d}\left(\begin{array}{c}
n-1 \\
i-1
\end{array}\right)(i-1) ! \cdot S(d-1, i-1) \sum_{j=i-1}^{d-1}\left(\begin{array}{c}
d-1 \\
j
\end{array}\right) \\
=\sum_{i=1}^{d}\left(\begin{array}{c}
n-1 \\
i-1
\end{array}\right) c_{i},
\end{gathered}
$$

where $c_{i}=(i-1) ! \cdot S(d-1, i-1) \sum_{j=i-1}^{d-1}\left(\begin{array}{c}d-1 \\ j\end{array}\right)$. The proof of Theorem 2. is completed.

\section{References}

[1] P. ERDős. On the distribution function of additive functions, Annals of Mathematics, 47 (1946), 1-20.

[2] P. ERdős, A. SÁRKözy. Problems and results on additive properties of general sequences I., Pacific Journal, 118 (1985), 347-357.

[3] P. ERdős, A. SÁrközy. Problems and results on additive properties of general sequences II., Acta Mathematica Hungarica, 48 (1986), 201-211. 
[4] P. Erdős, A. SÁrközy, V. T. Sós. Problems and results on additive properties of general sequences III., Studia Scientiarum Mathematicarum Hungarica, 22 (1987), 53-63.

[5] P. Erdős, A. SÁrközy, V. T. Sós. Problems and results on additive properties of general sequences IV., in: Number Theory, Proceedings, Ootacamund, India, 1984, Lecture Notes in Mathematics 1122, Springer-Verlag, (1985), 85-104.

[6] P. ERdős, A. SÁRközy, V. T. Sós. Problems and results on additive properties of general sequences $V$., Pacific Journal, 22 (1987), 53-63.

[7] G. Grekos, L. Haddad, C. Helou, J. Pihko. Representation functions, Sidon sets and bases, Acta Arithmetica, 130 (2007), 149-156.

[8] P. Minailescu. A class number free criterion for Catalan's conjecture, Journal of Number Theory, 99 (2003), 225-231.

[9] I. Niven, H. S. Zuckerman, H. L. Montgomery. An Introduction to The Theory of Numbers, 5th ed., Wiley, 1991.

[10] P. ERdős, P. TuRÁn. On a problem in the elementary theory of numbers, American Mathematical Monthly, 41 (1934), 608-611.

[11] A. SÁRKÖzY. On the number of additive representations of integers, in: More Sets, Graphs and Numbers, A Salute to Vera T. Sós and András Hajnal, eds. E. Györy et al., Bolyai Soc. Math. Studies, 15, Conference on Finite and Infinite Sets, 2006, J. Bolyai Math. Soc. and Springer; 329-339.

[12] A. SÁrközy, V. T. Sós. On Additive representative functions, in: The Mathematics of Paul Erdős, Edited by R. L. Graham, J. Nesetril and Steve Butler., Springer, New York, 2013,

[13] R. P. Stanley. Enumerative Combinatorics, Vol. 1. Second ed. Cambridge Studies in Advanced Mathematics, 49, Cambridge University Press, Cambridge, 2012. 